\title{
Einstein's Real "Biggest Blunder"
}

\author{
Homer G. Ellis \\ Department of Mathematics \\ University of Colorado Boulder \\ Boulder, Colorado 80309-0395 \\ Email: ellis@euclid.colorado.edu
}

November 6, 2018

\begin{abstract}
Albert Einstein's real "biggest blunder" was not the 1917 introduction into his gravitational field equations of a cosmological constant term $\Lambda$, rather was his failure in 1916 to distinguish between the entirely different concepts of active gravitational mass and passive gravitational mass. Had he made the distinction, and followed David Hilbert's lead in deriving field equations from a variational principle, he might have discovered a true (not a cut and paste) Einstein-Rosen bridge and a cosmological model that would have allowed him to predict, long before such phenomena were imagined by others, inflation, a big bounce (not a big bang), an accelerating expansion of the universe, dark matter, and the existence of cosmic voids, walls, filaments, and nodes.
\end{abstract}

Albert Einstein is reputed to have said that his "biggest blunder" was the 1917 introduction into his gravitational field equations of a cosmological constant term $\Lambda$ [1, 2]. In retrospect this looks to have been closer to an unwitting act of prescience than to a blunder, as $\Lambda$ seems to offer a route to understanding (in a superficial way) the acceleration of the expansion of the universe.

What has gone unrecognized for almost a century is that already in 1916 Einstein had made a real blunder by failing to distinguish between the entirely different concepts of active gravitational mass and passive gravitational mass. That he confused the two becomes clear upon a careful reading of $\S 16$ of his paper Die Grundlage der allgemeinen Relativitätstheorie [3], in which he sought to extend his tensorial field equations for empty space to the case in which space is permeated by a continuous distribution of gravitating matter, in analogy to the extension of the Laplace equation $\nabla^{2} \phi=0$ for the vacuum gravitational potential $\phi$ of newtonian gravity to the Poisson equation $\nabla^{2} \phi=4 \pi \kappa \mu$, where $\kappa$ is Newton's gravitational constant and $\mu$ denotes, in Einstein's imprecise choice of words, the "density of matter". Whatever he had in mind when he wrote that phrase, it is clear that $\mu$ is the active (gravitating), not the passive (gravitated), mass density of the matter in question.

The (weak) principle of equivalence, which Einstein had specifically built into his general theory of relativity, identifies passive gravitational mass with inertial mass, and the special theory identifies inertial mass with energy via $E=m c^{2}$. When Einstein, continuing in $\S 16$, wrote that (in the special theory) "energy... finds its complete mathematical expression in a tensor of the second rank, the energy-tensor", and then, relying on these identifications, proceeded to introduce as the material source term in his field equations "a corresponding energy-tensor of matter $\mathrm{T}_{\sigma}^{\alpha}$ ", he implicitly assumed, without presenting any justification for it, that passive gravitational mass could play the same role in his equations that active gravitational mass plays in the Poisson equation of newtonian gravity. That was his real "biggest blunder".

It is a reasonable speculation that Einstein was seduced into this logical error by the fact that in newtonian gravity application of the law of action and reaction to the forces exerted on each 
other by two gravitating bodies $\mathrm{A}$ and $\mathrm{B}$ allows the inference that the ratio of active mass to passive mass is the same for B as it is for A, thus by extension is the same for all such material bodies. If he was guided by this, whether consciously or unconsciously, then he was misguided, for this application of the action-reaction law to bodies not in contact requires the assumption that gravity acts instantaneously over the intervening distance, an assumption at odds with the finiteness of the propagation velocity of gravitational effects implicit in Einstein's theory.

Whatever caused Einstein to make the error, it was solidified in his mind by a consequence he asserted to be "the strongest reason for the choice" of his "energy-tensor" source term for a "frictionless adiabatic fluid" of "density" $\rho$, pressure $p$, and proper four-velocity distribution $u^{\alpha}$. The choice he made was $T^{\alpha \beta}=\rho u^{\alpha} u^{\beta}-\left(p / c^{2}\right) g^{\alpha \beta}$ (revised to $T^{\alpha \beta}=(\rho+p) u^{\alpha} u^{\beta}-\left(p / c^{2}\right) g^{\alpha \beta}$ by his redefintion $\rho \rightarrow \rho+p)$. The consequence he liked was that the vanishing of the divergence of the left member of the resulting field equation $\boldsymbol{R}^{\alpha \beta}-\frac{1}{2} \boldsymbol{R} g^{\alpha \beta}=\frac{8 \pi \kappa}{c^{2}} T^{\alpha \beta}$ (in today's notation) implied the vanishing of the divergence $T^{\alpha \beta}: \beta$ of $T^{\alpha \beta}$, which, interpreted as "the Eulerian hydrodynamical equations of the general theory of relativity", ... "give a complete solution of the problem of motion" $([3],\{19)$. That would certainly be an alluring consequence if it were not based on the confusion between the inertial-passive mass density $\rho$ and the active gravitational mass density $\mu$ of the fluid in question.

Had Einstein recognized the error implicit in making the "energy-tensor of matter $\mathrm{T}_{\sigma}^{\alpha}$ " the material source term in his field equations, what road might he then have followed? Having already incorporated into his theory a variational principle to identify worldlines of test particles as geodesic paths of the space-time metric, he might have looked for a variational principle from which to derive field equations. That is what David Hilbert did contemporaneously to derive field equations with the electromagnetic field providing the source term (field equations which for the vacuum matched those of Einstein, giving rise to a still ongoing debate over assignment of priority) 44. What neither Einstein nor Hilbert thought to do was look for inspiration to the variational principle from which the Poisson equation for $\phi$ derives, namely,

$$
\delta \int\left(|\nabla \phi|^{2}+8 \pi \kappa \mu \phi\right) d^{3} x=0 .
$$

From Hilbert's variational principle it would have been straightforward to arrive at the generalization

$$
\delta \int\left(\boldsymbol{R}-\frac{8 \pi \kappa}{\mathrm{c}^{2}} \mu\right)|g|^{\frac{1}{2}} d^{4} x=0
$$

of (11), for which the Euler-Lagrange equations are equivalent to

$$
\boldsymbol{R}^{\alpha \beta}-\frac{1}{2} \boldsymbol{R} g^{\alpha \beta}=-\frac{4 \pi \kappa}{\mathrm{c}^{2}} \mu g^{\alpha \beta},
$$

equivalent in turn to $\boldsymbol{R}_{\alpha \beta}=\frac{4 \pi \kappa}{c^{2}} \mu g_{\alpha \beta}$, the 00 component of which reduces in the slowly varying, weak field approximation to the Poisson equation, with $\phi=\frac{1}{2}\left(g_{00}-c^{2}\right)$. This would have had the salutary effect of replacing Einstein's unjustified "energy-tensor" source term by $T^{\alpha \beta}=-\frac{4 \pi \kappa}{c^{2}} \mu g^{\alpha \beta}$, properly built with $\mu$ instead of $\rho$. A not so salutary effect is that the vanishing of the divergerce of $T^{\alpha \beta}$ would imply that $\mu$ must be constant, which would suggest that the only cosmological models that could be solutions of (3) would be the discredited 'steady-state' models. A way out of this dilemma will present itself below.

Let us suppose that Einstein had arrived at (3) by way of the variational principle (2), and published that instead of the equations he did publish. How might subsequent developments in the general theory of relativity have played out? No doubt Schwarzschild would have derived the same well-known blackhole metric he published in 1916, for the vacuum equations are the same, but when he searched for an interior solution to go with it he would have found a model for the gravitational field inside a star simpler and more understandable than the one he published as his 'interior solution' of Einstein's original equations [5]. What else? 
We know that in 1935 Einstein and Rosen tried to construct a singularity-free model of an elementary particle as a 'bridge' (later termed a 'wormhole') connecting two copies of the region of Schwarzschild's blackhole outside the $r=2 m$ horizon, but could do so only by weakening the field equations in a mathematically suspect manner (replacing $\boldsymbol{R}_{\alpha \beta}$ with $g^{2} \boldsymbol{R}_{\alpha \beta}$, where $g$ is the determinant of the metric tensor) [6]. Because their method would not work if $m$ were negative, they believed that they had found a reason why such a particle could have only a positive or a zero inertial mass, once again confusing active gravitational mass (the $m$ of the Schwarzschild blackhole) with the conceptually distinct inertial-passive mass. Now let us imagine that Einstein (with or without Rosen), instead of contenting himself with this ad hoc cut and paste job, had investigated Schwarzschild's solution more thoroughly and had come across one of the papers by Gullstrand 7 and Painlevé [8] that expressed Schwarzschild's blackhole metric in the form $d t^{2}-(d r-v d t)^{2}-r^{2} d \Omega^{2}$, where $v=-\sqrt{\frac{2 m}{r}}$, the velocity of an observer free-falling from rest at $r=\infty$. He might then have noticed that to such an observer space, in the sense of a $t=$ constant slice of space-time, would have no curvature, its metric being that of euclidean three-space in spherical coordinates, namely, $d r^{2}+r^{2} d \Omega^{2}$. Studying the matter further he could have realized that such curvature would be admitted if the field equations were weakened by intoduction of a scalar field $\psi$ minimally coupled to the space-time geometry. This would be accomplished by modifying the integrand of the action integral of (2) to $\boldsymbol{R}-\frac{8 \pi \kappa}{c^{2}} \mu-2 \psi^{\cdot \gamma} \psi_{\cdot \gamma}$. But this new, enlightened Einstein would not stop there, for having recognized that 'energy', if coupled to geometry at all, need not be coupled with the same polarity that ordinary matter is coupled with, and, seeing no reason to choose one polarity over the other, would use a second scalar field $\phi$ (not the newtonian $\phi$ ) to further modify the integrand to $\boldsymbol{R}-\frac{8 \pi \kappa}{c^{2}} \mu+2 \phi^{\cdot \gamma} \phi_{. \gamma}-2 \psi^{\cdot \gamma} \psi_{. \gamma}$. Moreover, the new Einstein, holding just as the old to the principle that all things physical were to be found embedded intrinsically in the geometry of space-time, would derive new field equations from the thus modified variational principle by varying only the metric components, and not the scalar fields (or for that matter, the density $\mu$ ). The field equations that would have resulted are

$$
\boldsymbol{R}_{\alpha \beta}-\frac{1}{2} \boldsymbol{R} g_{\alpha \beta}=-\frac{4 \pi \kappa}{c^{2}} \mu g_{\alpha \beta}-2\left(\phi_{. \alpha} \phi_{. \beta}-\frac{1}{2} \phi^{\cdot \gamma} \phi_{. \gamma} g_{\alpha \beta}\right)+2\left(\psi_{. \alpha} \psi_{. \beta}-\frac{1}{2} \psi^{\cdot \gamma} \psi_{. \gamma} g_{\alpha \beta}\right) .
$$

Pressing on, Einstein (perhaps with the help of Rosen) might well have arrived in 1935 at the true Einstein-Rosen bridge first discovered around 1970 independently of one another by two researchers, and called descriptively by one of them a 'drainhole' with a gravitational 'ether' flowing through it [9, 10. This geodesically complete, nonsimply connected space-time manifold lacks both the singularity and the event horizon of the Schwarzschild blackhole. It is specified by assignment of two parameters: $m$, the active gravitational mass of the drainhole as measured at infinity on the 'high' side, and $n$, which determines the size of the drainhole, subject only to the constraints that $0 \leq m<n$. Had he dug far enough, Einstein would have found that, because the 'ether', describable as a cloud of test particles free-falling from rest at infinity on the high side (or simply as space itself flowing from the high side to the low side), accelerates downward all the way into and through the drainhole and out to minus infinity on the low side, the drainhole gravitationally attracts all matter on its high side but repels all matter on the low side. Digging even deeper he would have learned that the negative mass parameter $\bar{m}$ specifying the strength of the repulsion on the low side exceeds in magnitude the attractive mass parameter $m$ in the ratio $-\bar{m} / m=e^{m \pi / \sqrt{n^{2}-m^{2}}}$. An inevitable conclusion from this would be that in addition to the positive mass density $\mu$ there must exist a negative mass density $\bar{\mu}$, thus an overall negative net active gravitational mass density $\mu+\bar{\mu}$ to replace the $\mu$ in (4), turning it into

$$
\boldsymbol{R}_{\alpha \beta}-\frac{1}{2} \boldsymbol{R} g_{\alpha \beta}=-\frac{4 \pi \kappa}{c^{2}}(\mu+\bar{\mu}) g_{\alpha \beta}-2\left(\phi_{. \alpha} \phi_{. \beta}-\frac{1}{2} \phi^{\cdot \gamma} \phi_{. \gamma} g_{\alpha \beta}\right)+2\left(\psi_{. \alpha} \psi_{. \beta}-\frac{1}{2} \psi^{\cdot \gamma} \psi_{. \gamma} g_{\alpha \beta}\right) .
$$

Having gone this far, the new Einstein would surely have 'divergenced' this equation and arrived at

$$
2(\square \phi) \phi_{. \alpha}-2(\square \psi) \psi_{. \alpha}:=2 \phi_{: \gamma}^{\cdot \gamma} \phi_{. \alpha}-2 \psi_{: \gamma}^{\cdot \gamma} \psi_{. \alpha}=-\frac{4 \pi \kappa}{c^{2}}(\mu+\bar{\mu})_{. \alpha},
$$


which he could have recognized as a weaker replacement for the equations $\square \phi=0$ and $\square \psi=0$ that varying $\phi$ and $\psi$ would have produced, and which would have required neither $\mu$, nor $\bar{\mu}$, nor their net $\mu+\bar{\mu}$ to be constant.

The new, enlightened Einstein might well have introduced in 1917 a cosmological constant $\Lambda$, for the same reasons that the old Einstein relied on, and after Hubble's discovery that the universe is expanding, consigned it to the trash bin just as before. But after 1935 he might well also have revisited the question, seen that his cosmological constant was in effect the negative net density $\mu+\bar{\mu}$ in disguise, and retracted his characterization of it as his "biggest blunder" (thus reducing his "biggest blunder" count to zero). Investigating the cosmological consequences of (5) he might easily have discovered the model of the universe presented in [11, and thereby have predicted, long before such phenomena were imagined by others, inflation, a big bounce (not a big bang), an accelerating expansion of the universe, dark matter, and the existence of cosmic voids, walls, filaments, and nodes.

All of that is only a small part of what might have transpired had Einstein recognized his real "biggest blunder" and followed the road that then would have stretched out before him. For those who admire and respect the work that he did it should be a cause for real regret that in the long and arduous process of constructing his general theory of relativity he missed this opportunity to make the theory logically consistent and therefore more powerful.

\section{References}

[1] A. Einstein, Kosmologische Betrachtungen zur allgemeinen Relativitätstheorie, Sitzungsber. Preuss. Akad. Wiss. phys.-math. Klasse VI (1917), 142-152; translated in The Principle of Relativity (Dover, New York, 1952), pp. 177-188.

[2] G. Gamow, My World Line: An Informal Autobiography, (Viking Press, New York, 1970), 44.

[3] A. Einstein, Die Grundlage der allgemeinen Relativitätstheorie, Ann. der Physik 49 (1916), 769-822; translated in The Principle of Relativity (Dover, New York, 1952), pp. 109-164.

[4] D. Hilbert, Die Grundlagen der Physik (Erste Mitteilung), Nachr. Königl. Gesellschaft d. Wiss. Göttingen, Math.-Phys. Kl., (1916), 395-407; reprised in Math. Annalen 92 (1924), 1-32.

[5] H. G. Ellis, Gravity inside a nonrotating, homogeneous, spherical body, arxiv:gr-qc/1203.4750 [gr-qc] (2012).

[6] A. Einstein and N. Rosen, The particle problem in the general theory of relativity, Phys. Rev. 48 (1935), 73-77.

[7] A. Gullstrand, Allgemeine Lösung des statischen Einkörperproblems in der Einsteinschen Gravitationstheorie, Arkiv. Mat. Astron. Fys. 16 (1922), 1-15.

[8] P. Painlevé, La mécanique classique et la théorie de la relativité, C. R. Hebd. Acad. Sci. (Paris) 173 (1921), 677-680.

[9] H. G. Ellis, Ether flow through a drainhole: A particle model in general relativity, J. Math. Phys. 14 (1973), 104-118; Errata: 15 (1974), 520.

[10] K. A. Bronnikov, Scalar-tensor theory and scalar charge, Acta Phys. Pol. B4 (1973), 251-266.

[11] H. G. Ellis, Cosmic inflation, deceleration, acceleration, dark matter, and dark 'energy' in one coherent package, arxiv:gr-qc/0701012 (2011). 